\title{
Type I interferons suppress viral replication but contribute to $T$ cell depletion and dysfunction during chronic HIV-1 infection
}

\author{
Liang Cheng, ${ }^{1}$ Haisheng Yu, ${ }^{2}$ Guangming Li, ${ }^{1}$ Feng Li ${ }^{1,3}$ Jianping Ma, ${ }^{2}$ Jingyun Li, ${ }^{2}$ Liqun Chi, ${ }^{1}$ \\ Liguo Zhang, ${ }^{2}$ and Lishan Su, ${ }^{1,24}$ \\ 'Lineberger Comprehensive Cancer Center, University of North Carolina at Chapel Hill, Chapel Hill, North Carolina, USA. \\ ${ }^{2}$ Key Laboratory of Infection and Immunity, Institute of Biophysics, Chinese Academy of Sciences, Beijing, China. \\ ${ }^{3}$ Institute of Infectious Diseases, Guangzhou Eighth People's Hospital, Guangzhou Medical University, Guangzhou, \\ Guangdong, China. ${ }^{4}$ Department of Microbiology and Immunology, University of North Carolina at Chapel Hill, Chapel Hill, \\ North Carolina, USA.
}

The direct link between sustained type I interferon (IFN-I) signaling and HIV-1-induced immunopathogenesis during chronic infection remains unclear. Here we report studies using a monoclonal antibody to block IFN- $\alpha / \beta$ receptor 1 (IFNAR1) signaling during persistent HIV-1 infection in humanized mice (hu-mice). We discovered that, during chronic HIV-1 infection, IFNAR blockade increased viral replication, which was correlated with elevated T cell activation. Thus, IFN-Is suppress HIV-1 replication during the chronic phase but are not essential for HIV-1-induced aberrant immune activation. Surprisingly, IFNAR blockade rescued both total human T cell and HIVspecific T cell numbers despite elevated HIV-1 replication and immune activation. We showed that IFNAR blockade reduced HIV-1-induced apoptosis of CD4+ $\mathrm{T}$ cells. Importantly, IFNAR blockade also rescued the function of human T cells, including HIV-1-specific CD8 ${ }^{+}$and CD4 ${ }^{+} \mathrm{T}$ cells. We conclude that during persistent HIV-1 infection, IFN-Is suppress HIV-1 replication, but contribute to depletion and dysfunction of T cells.

Conflict of interest: The authors have declared that no conflict of interest exists.

Submitted: April 4, 2017 Accepted: May 10, 2017 Published: June 15, 2017

\section{Reference information:} JCI Insight. 2017;2(12):e94366. https://doi.org/10.1172/jici. insight.94366.

\section{Introduction}

Type I interferons (IFN-Is) are critical for controlling various viral infections $(1,2)$, but the precise role of IFN-Is during chronic HIV-1 infection is still not clearly defined (3, 4). HIV-1 infection induces sustained expression of IFN-Is $(5,6)$. It has been shown that IFN-Is can suppress HIV-1 replication in vitro (3). IFN-Is have also been shown to inhibit initial simian immunodeficiency virus (SIV) infection in rhesus macaques (7) and HIV-1 infection in humanized mice $(8,9)$ in vivo. Furthermore, reports also indicated that transmitted/founder viruses isolated from patients are less sensitive to suppression by IFN- $\alpha$ in cultured cells than their corresponding viruses present during chronic infection, suggesting that resistance to IFN-Imediated inhibition may provide a selective advantage during transmission and initial infection $(10,11)$. These observations suggest that a robust IFN-I response helps to control early HIV-1 and SIV infection.

The contribution of IFN-Is to the control of HIV-1 replication and to the immunopathogenesis of AIDS during chronic infection is under debate $(3,4)$. Recently, studies using nonhuman primate models show that sustained IFN-I signaling is associated with pathogenic SIV infection. IFN-Is are equally induced during acute SIV infection in both nonpathogenic (African green monkeys or sooty mangabeys) and pathogenic (rhesus macaques or pigtail macaques) hosts. However, compared with the nonpathogenic infection, pathogenic SIV infection of rhesus macaques leads to AIDS correlating with persistent IFN-I signaling (12-15). These studies imply that there is a correlation between IFN-I signaling and SIV-induced pathogenesis, although it is unclear whether the ability of the natural hosts to shut off the IFN-I response directly contributes to the outcomes or is a secondary effect. Supportively, in persistent lymphocytic choriomeningitis virus (LCMV) infection in mice, blocking IFN-I signaling can reduce immune activation, diminish immune suppression, restore lymphoid architecture, and accelerate clearance of the virus (16-18). 
A

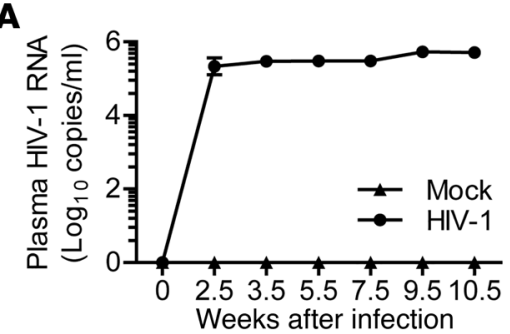

B

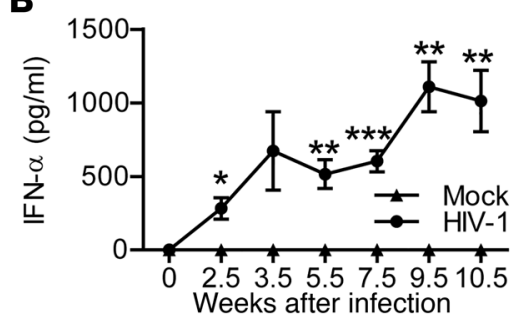

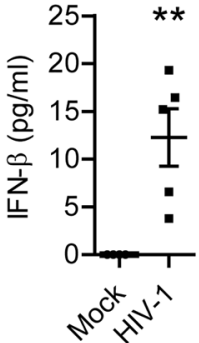
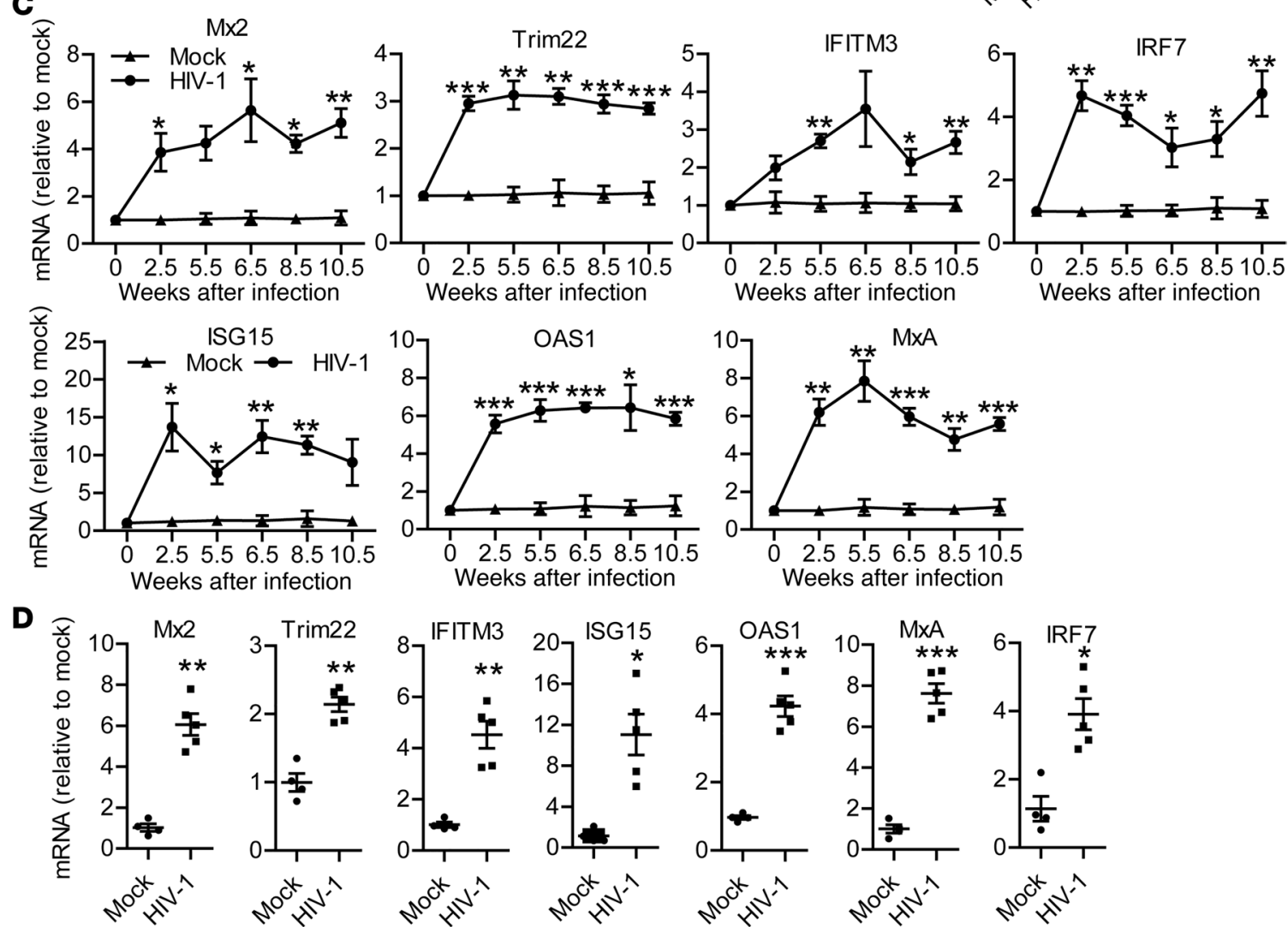

Figure 1. Persistent HIV-1 infection in humanized mice leads to sustained and systemic type I IFN expression and IFN-stimulated gene induction.

Humanized mice were infected with HIV-1. (A) Plasma HIV-1 genomic RNA levels at indicated time points. (B) Human IFN- $\alpha$ (pan IFN- $\alpha$ ) levels in plasma at indicated time points and IFN- $\beta$ levels in plasma at 10.5 weeks post infection (wpi). (C) Relative mRNA levels of Mx2, IFITM3, Trim22, ISG15, OAS1, MxA, and IRF7 in peripheral blood mononuclear cells at indicated time points. (D) The relative mRNA levels of Mx2, IFITM3, Trim22, ISG15, OAS1, MxA, and IRF7 in spleens at termination (10.5 wpi). Shown are representative data (mock, $n=4$; HIV-1, $n=5$ ) of 4 independent experiments (mock, $n=12 ;$ HIV-1, $n=18$ in total) with mean values $\pm \mathrm{SEM}$. ${ }^{*} P<0.05,{ }^{* *} P<0.01,{ }^{* *} P<0.001$. Unpaired, 2-tailed Student's $t$ test was performed to compare between groups at singular time points (B and $\mathbf{C}$ ) or between 2 groups (D).

Administration of exogenous IFN- $\alpha$ was reported to lower HIV-1 burden but failed to show a significant benefit in HIV-1 disease progression in HIV-1-infected patients (4). Association studies in HIV-1-infected patients indicated that overexpression of IFN-Is and interferon-stimulated genes (ISGs) is correlated with higher levels of viral load, enhanced hyper-immune activation, and faster rates of disease progression $(6,19-21)$. These reports highlight that IFN-Is may play important but complex roles in HIV-1 infection and pathogenesis.

A study by Sandler et al. reported that during the acute phase (0-4 weeks after infection) of SIV infection, blocking IFN-I signaling with an antagonistic mutant recombinant IFN- $\alpha 2$ (IFN-ant), with increased IFN$\alpha / \beta$ receptor 2 (IFNAR2) binding but diminished IFNAR1 binding activity (22), leads to elevated SIV replication and accelerated disease progression in rhesus monkeys (7). However, this report only defined the role of IFN-Is during acute SIV infection. The role of IFN-Is during chronic SIV infection is still unclear.

To clarify the causal link between sustained IFN-I signaling and HIV-1-induced immunopathogenesis, we developed a mAb against IFNAR1 to block IFN-I signaling during persistent HIV-1 infection in 
A
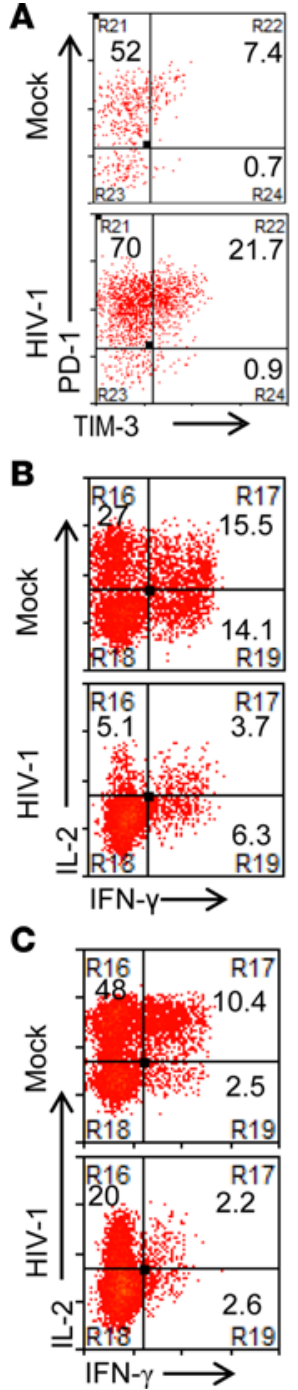
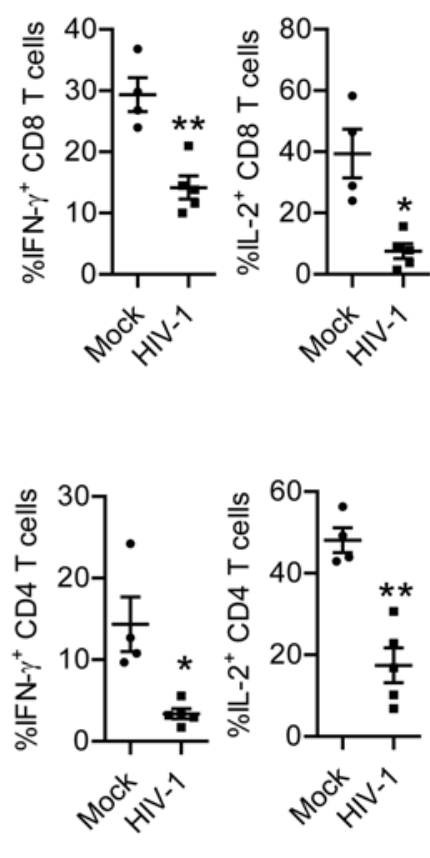

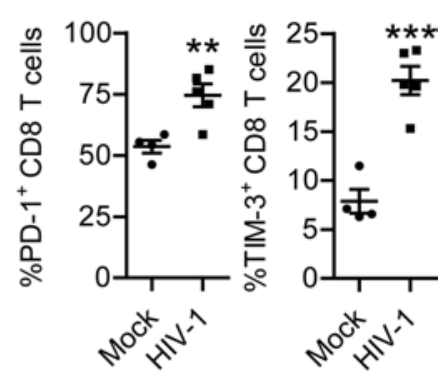

Figure 2. Persistent HIV-1 infection in humanized mice leads to impaired T cell function. Humanized mice were infected with HIV-1 and terminated at 10.5 weeks postinfection (wpi). (A) Representative dot plots and summarized data show the percentage PD- $1^{+}$and $\mathrm{TIM}-3^{+}$of $\mathrm{CD} 8^{+}$T cells in spleens. (B and C) At $10.5 \mathrm{wpi}$, splenocytes were stimulated ex vivo with PMA/ionomycin for 4 hours followed by intracellular cytokine staining. (B) Representative dot plots (of cells gated on human $\left.\mathrm{CD}_{4}{ }^{+} \mathrm{CD3}^{+} \mathrm{CD}^{+}\right)$and summarized data show the percentages of IFN- $\gamma$-producing and IL-2-producing CD8 ${ }^{+} \mathrm{T}$ cells. (C) Representative dot plots (of cells gated on human $\left.\mathrm{CD} 45^{+} \mathrm{CD}^{+} \mathrm{CD}^{+}\right)$and summarized data show the percentages of IFN- $\gamma$-producing and IL-2-producing CD4 ${ }^{+}$T cells. Shown are representative data (mock, $n=4$; HIV-1, $n=5$ ) of 4 independent experiments (mock, $n=12$; HIV-1, $n=18$ in total) with mean values \pm SEM. ${ }^{*} P<0.05,{ }^{* *} P<0.01,{ }^{* *} P<0.001$ by unpaired, 2 -tailed Student's $t$ test.

humanized mice with a functional human immune system (hu-mice). We have reported that the anti-IFNAR1 mAb specifically binds human IFNAR1 and inhibits human IFN-I activity both in vitro and in vivo (23). We found that IFNAR blockade during persistent HIV-1 infection rescued human T cells both numerically and functionally, despite elevated HIV-1 replication and T cell activation. Thus, IFN-Is contribute to T cell depletion and dysfunction, as well as to innate virus control, during persistent HIV-1 infection.

\section{Results}

Persistent HIV-1 infection leads to sustained and systemic IFN-I induction. To functionally define the role of IFN-Is in persistent HIV-1 infection and pathogenesis, we employed hu-mice for modeling HIV-1 infection and immunopathogenesis (24-26). HIV-1 infection in hu-mice resulted in sustained plasma viremia (Figure 1A). Similar to clinical observations (4, 27), HIV-1 infection in hu-mice also led to induction of IFN-Is including IFN- $\alpha$ and IFN- $\beta$ (Figure 1B) and the induction of ISGs, including Mx2, IFITM3, Trim22, ISG15, OAS1, MxA, and IFN regulatory factor 7 (IRF7) both in peripheral blood mononuclear cells (PBMCs) (Figure 1C) and in the spleen (Figure 1D).

As observed in HIV-1-infected patients, HIV-1 infection in hu-mice induced severe depletion of human leukocytes including $\mathrm{CD} 4^{+} \mathrm{T}$ cells in both peripheral blood and lymphoid organs (Supplemental Figure 1, A-E; supplemental material available online with this article; https://doi. org/10.1172/jci.insight.94366DS1). Persistent HIV-1 infection also led to aberrant T cell activation, as indicated by enhanced expression of HLA-DR, CD38, and Ki-67 (Supplemental Figure 1, F-I). Additionally, human T cells in HIV-1-infected mice showed increased expression of exhaustion markers PD-1 and TIM-3 (Figure 2A), which was associated with impaired T cell functions as indicated by decreased capacity to produce IFN- $\gamma$ and IL- 2 by both $\mathrm{CD} 8^{+}$and $\mathrm{CD} 4^{+} \mathrm{T}$ cells upon PMA/ionomycin stimulation (Figure 2, B and C). Therefore, persistent HIV-1 infection in hu-mice led to systemic and sustained IFN-I signaling associated with $\mathrm{CD} 4^{+} \mathrm{T}$ cell depletion, aberrant immune activation, and $\mathrm{T}$ cell exhaustion.

IFNAR1 blockade during persistent HIV-1 infection elevates HIV-1 replication. To define the role of IFN-Is during persistent HIV-1 infection in vivo, we infected hu-mice and treated them with IFNAR1-blocking $\mathrm{mAb}$ from 6 to 10 weeks postinfection (wpi). As expected, IFNAR1 mAb treatment blocked the expression of ISGs, including those with anti-HIV-1 function such as Mx2 $(28,29)$ and IFITM3 $(30,31)$, in PBMCs of infected hu-mice (Figure 3A and Supplemental Figure 2, A and B). We found that HIV-1 replication increased 8-fold within 1 week after IFNAR1 blockade and was sustained at higher levels than in control mice from 7 to 10 wpi (Figure 3B). IFNAR1 blocking resulted in higher IFN- $\alpha$ levels in peripheral blood (Figure 3C), which correlated with higher HIV-1 viremia in anti-IFNAR1-treated mice. At termination (10 wpi), IFNAR1 blockade also blocked ISG expression (Figure 3D and Supplemental Figure 2C) and increased HIV-1 replication in the spleen (Figure 3E). These results indicate that, during persistent HIV-1 infection, IFN-Is still contribute to the suppression of HIV-1 replication.

Elevated HIV-1 replication correlates with higher levels of $T$ cell activation and proinflammatory cytokine production after IFNAR1 blockade. We found that the frequencies of CD38/DR-positive and Ki-67-positive 


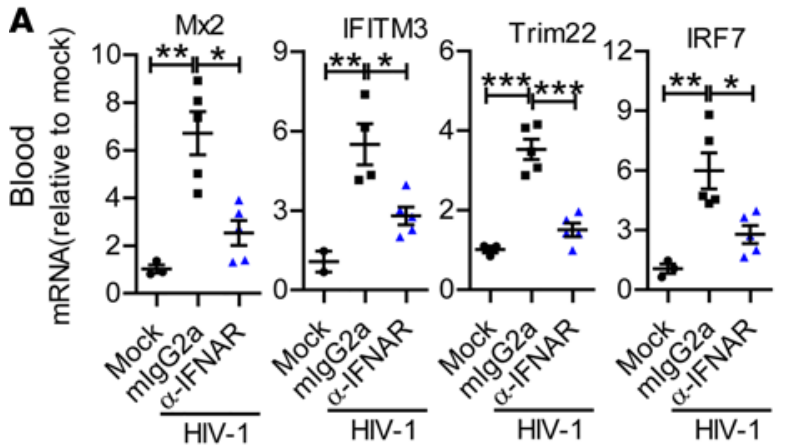

B

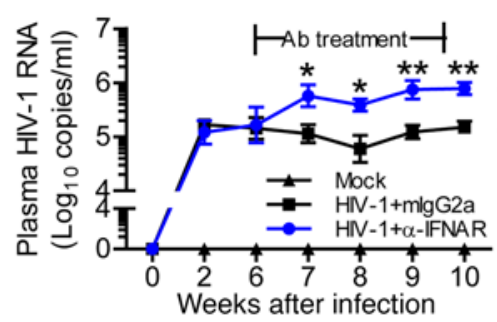

C

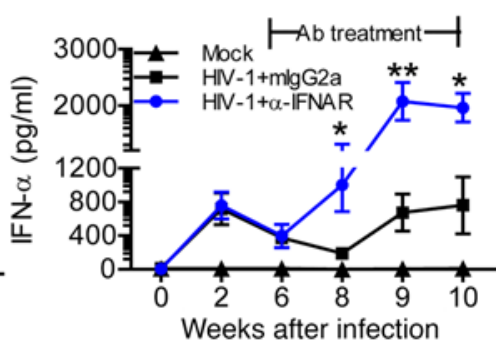

D
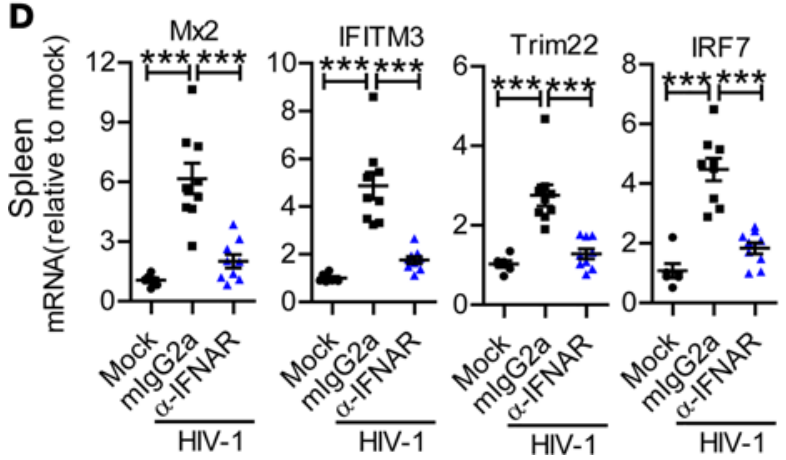

$\mathbf{E}$
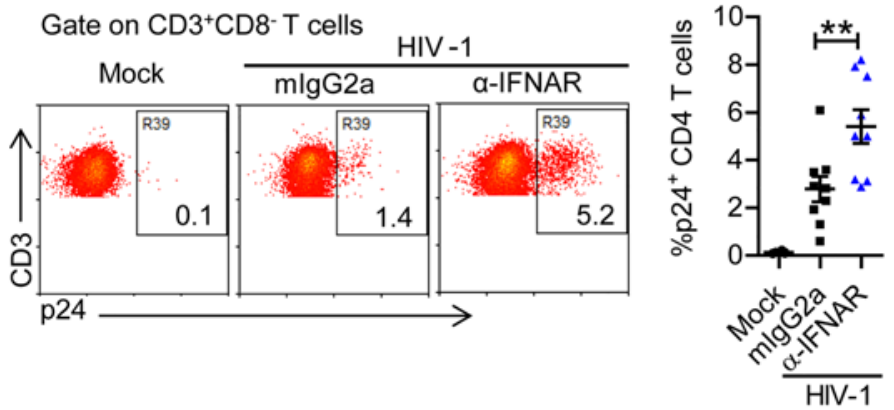

Figure 3. IFNAR1 blockade during persistent HIV-1 infection enhances viral replication in humanized mice. Humanized mice infected with HIV-1 were treated from 6 to 10 weeks postinfection (wpi) with a mAb against IFN- $\alpha / \beta$ receptor 1 (IFNAR1) or isotype control (mouse IgG2a) twice per week. (A) Relative mRNA levels of human interferon-stimulated genes including Mx2, IFITM3, TRIM22, and IRF7 in peripheral blood mononuclear cells at 9 wpi. (B) Plasma HIV-1 RNA levels at indicated time points after HIV-1 infection. (C) Human IFN- $\alpha$ levels in the plasma after HIV-1 infection. Shown (A-C) are representative data (mock, $n=3$; HIV-1 + mlgG2a, $n=5$; HIV-1 + anti-IFNAR1, $n=5$ ) of 3 independent experiments (mock, $n=7 ;$ HIV-1 + mIgG2a, $n=11 ;$ HIV- $1+$ anti-IFNAR1, $n=12$ in total) with mean values \pm SEM. (D) Relative mRNA levels of human Mx2, IFITM3, TRIM22, and IRF7 in splenocytes at 10 wpi. (E) Representative FACS plots and summarized data show the percentages of HIV-1 p24-positive CD4 ${ }^{+}$T cells (CD3+CD8-) in the spleen at 10 wpi. Shown (D and E) are combined data of 2 independent experiments (mock, $n=6$; HIV-1 + mlgG2a, $n=9$; HIV-1 + anti-IFNAR1, $n=9$ ) with mean values \pm SEM. ${ }^{*} P<0.05,{ }^{* *} P<0.01,{ }^{* * *} P<0.001 .0$ Oneway ANOVA and Bonferroni's post hoc test were performed to compare between groups at singular time points (B and $\mathbf{C}$ ) or between groups (A, D, and $\mathbf{E})$.

$\mathrm{CD}^{+}$and $\mathrm{CD}^{+} \mathrm{T}$ cells were increased by blocking IFNAR1 (Figure 4, A and B). The expression level of CD38/DR and Ki-67 on T cells was positively correlated with HIV-1 viremia in plasma (Figure 4, C and D). IFNAR1 blockade also led to increased expression of TNF- $\alpha$, MCP-1, IP-10, and IFN- $\gamma$ in plasma of HIV-1-infected hu-mice (Figure 4E). These results indicate that, in the absence of IFN-I signaling, elevated HIV-1 replication was correlated with higher immune activation. Thus, IFN-I signaling is not essential for the aberrant T cell immune activation during the chronic phase of HIV-1 infection.

IFNAR1 blockade during persistent HIV-1 infection rescues human T cells, including HIV-1-specific T cells. Systemic chronic immune activation is associated with $\mathrm{CD} 4^{+} \mathrm{T}$ cell depletion $(32,33)$. However, the causal link between immune activation and $\mathrm{CD}^{+} \mathrm{T}$ cell loss is unclear (32). We therefore analyzed the effect of IFNAR1 blockade on HIV-1-induced pathogenesis, including CD4 ${ }^{+} \mathrm{T}$ cell depletion. Surprisingly, despite increased HIV-1 replication and $\mathrm{T}$ cell activation, the number of human $\mathrm{CD}^{+} \mathrm{T}$ cells in the spleen and mesenteric lymph nodes (mLNs) was significantly increased in hu-mice with IFNAR1 blockade (Figure 5, A and $\mathrm{B})$. IFNAR1 blockade also rescued human $\mathrm{CD} 8^{+} \mathrm{T}$ cells and total human leukocytes in lymphoid organs (Figure 5, A and B). Furthermore, we found that IFNAR1 blockade significantly increased the percentage and number of HLA-A2/SL-9 (an epitope consisting of amino acids 77-85 of HIV-1 p17 protein) pentamerspecific CD8 ${ }^{+} \mathrm{T}$ cells in lymphoid organs (Figure 5, C and D, and Supplemental Figure 3). These results suggest that, in the absence of IFN-I signaling, elevated immune activation does not lead to T cell depletion. IFN-I signaling thus contributes to the depletion of T cells during persistent HIV-1 infection.

IFNAR1 blockade protects HIV-1-induced apoptosis of $C D 4^{+} T$ cells. Elevated apoptosis is correlated with $\mathrm{CD}^{+} \mathrm{T}$ cell depletion during HIV-1 infection (34-36). We detected higher levels of active caspase- 3 in $\mathrm{CD}^{+} \mathrm{T}$ cells after persistent HIV-1 infection in hu-mice (Figure 6A). IFNAR1 blockade reduced the level of active caspase- 3 in $\mathrm{CD}^{+} \mathrm{T}$ cells (Figure 6A). These results suggest that blockade of IFN-I signaling prevents HIV-1-induced $\mathrm{CD} 4^{+} \mathrm{T}$ cell apoptosis. 

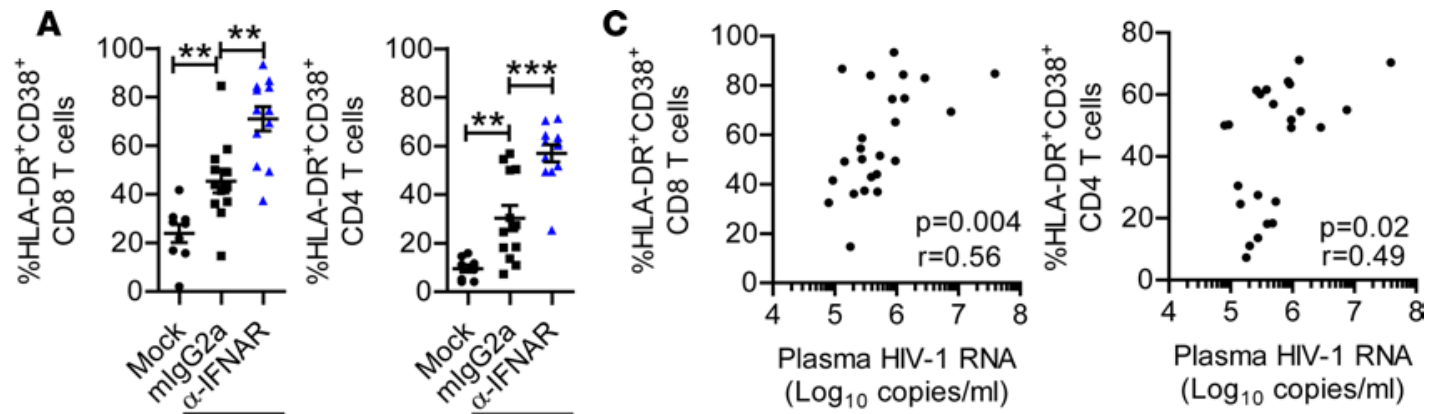

B
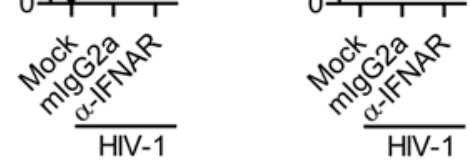

$\left(\log _{10}\right.$ copies/ml)

$\left(\log _{10}\right.$ copies $\left./ \mathrm{ml}\right)$
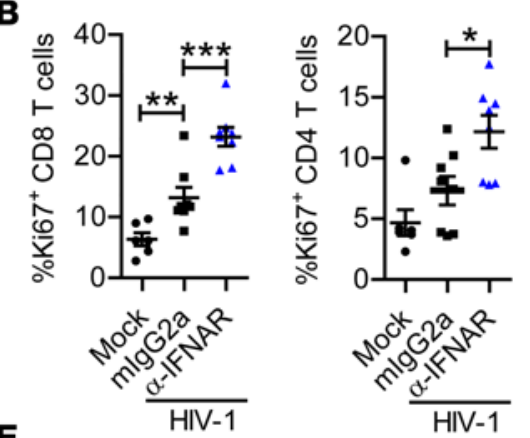

D
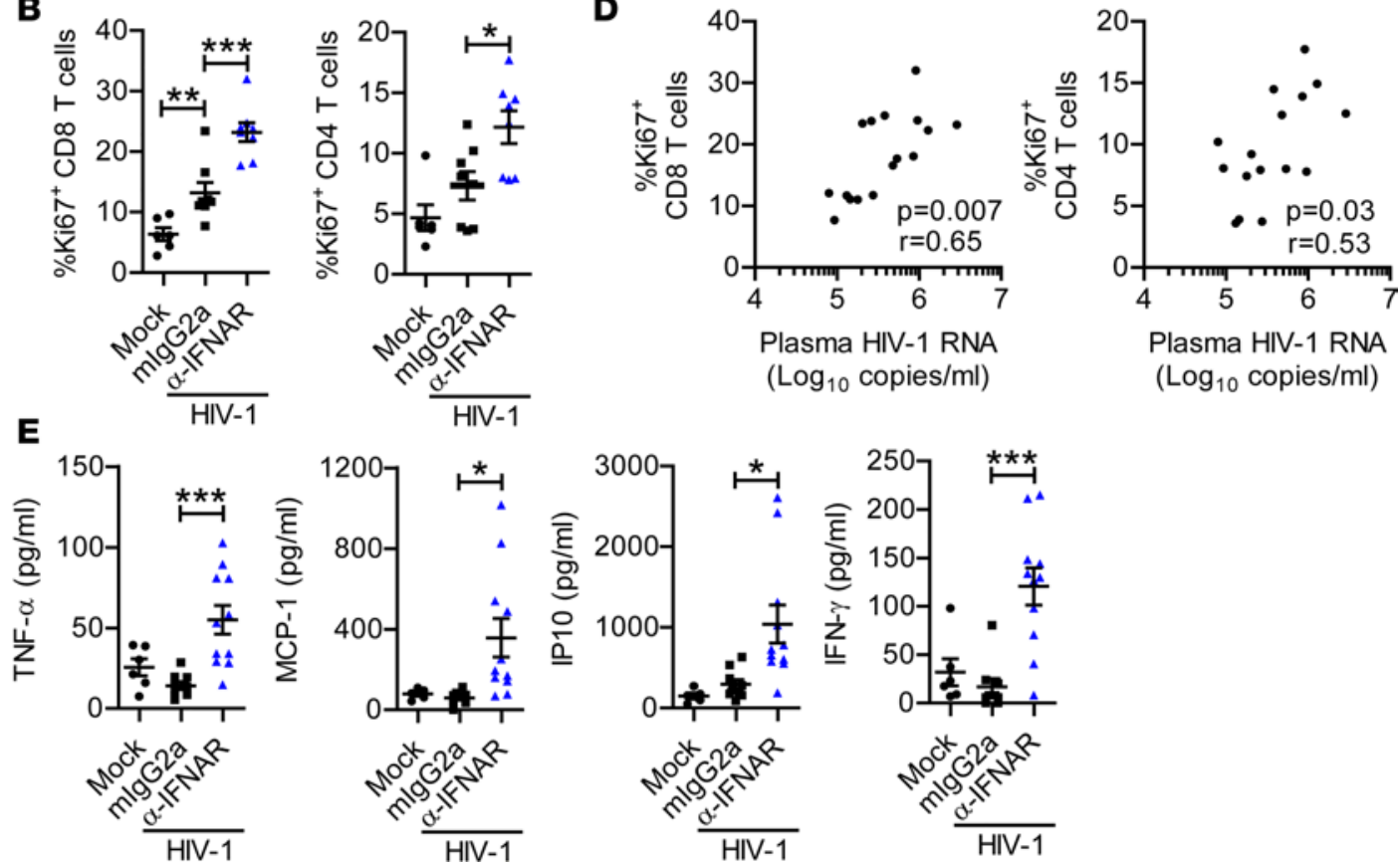

$\left(\log _{10}\right.$ copies/ml)

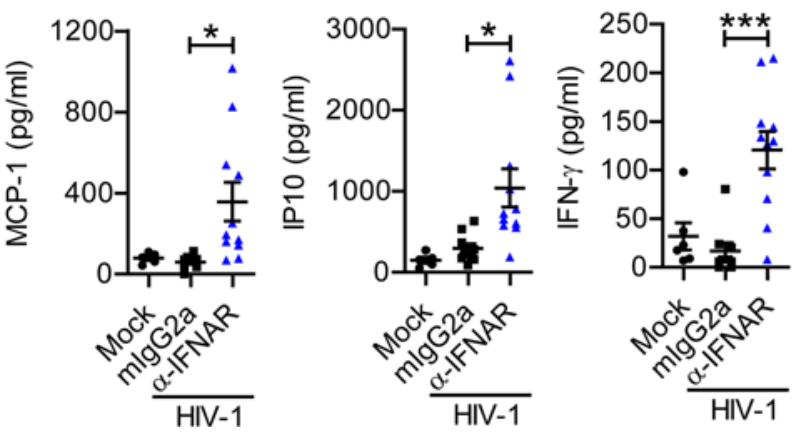

Figure 4. IFNAR1 blockade during persistent HIV-1 infection increases expression of HLA-DR/CD38 and Ki-67 on T cells. Humanized mice infected with HIV-1 were treated from 6 to 10 weeks postinfection (wpi) with a mAb against IFN- $\alpha / \beta$ receptor 1 (IFNAR1) or isotype control (mouse IgG2a) twice per week. Mice were sacrificed at 10 wpi. (A) Summarized data show CD8 ${ }^{+}$and CD4 ${ }^{+}$T cells expressing HLA-DR/CD38 (mock, $n=9$; HIV-1 + mlgG2a, $n=12$; HIV-1 + anti-IFNAR1, $n=12$, combined data from 3 independent experiment with mean values \pm SEM). (B) Summarized data show CD8 ${ }^{+}$and CD4 ${ }^{+}$T cells expressing Ki-67 (mock, $n=6$; HIV-1 + mIgG2a, $n=8$; HIV-1 + anti-IFNAR1, $n=8$, combined data from 2 independent experiments with mean values \pm SEM). (C) Correlation analysis between HLA-DR/CD38 expression on T cells and plasma HIV-1 RNA levels. (D) Correlation analysis between Ki-67 expression on T cells and plasma HIV-1 RNA levels. (E) The levels of indicated cytokines in the plasma of humanized mice at 10 wpi. Shown (E) are combined data of 3 independent experiments (mock, $n=6$; HIV-1 + mIgG2a, $n=9$; HIV-1 + anti-IFNAR1, $n=11$ ) with mean values \pm SEM. ${ }^{*} P<0.05,{ }^{* *} P<0.01,{ }^{* * *} P<0.001$ by 1-way ANOVA and Bonferroni's post hoc test (A, B, and $\mathbf{E})$ and Spearman rank correlation test (C and $\mathbf{D})$.

We then used an ex vivo culture system to further investigate how IFN-I signaling leads to CD4 ${ }^{+} \mathrm{T}$ cell depletion (37). We isolated splenocytes from hu-mice persistently infected with HIV-1 and cultured them ex vivo in the presence of isotype control $\mathrm{Ab}$, anti-IFNAR1 mAb, or IFN- $\alpha$. IFNAR1 blockade increased HIV-1 replication and exogenous IFN- $\alpha$ inhibited HIV-1 replication (Figure 6B). We found that, as in lymphoid organs in vivo, $\mathrm{CD}^{+} \mathrm{T}$ cells from HIV-infected mice showed higher levels of active caspase- 3 (Figure 6, C and D). IFNAR1 blockade reduced the level of active caspase- 3 in $\mathrm{CD}^{+} \mathrm{T}$ cells (Figure 6 , $\mathrm{C}$ and D). Accordingly, exogenous IFN- $\alpha$ added to the culture system further increased the level of active caspase-3 (Figure 6, C and D). Thus, IFNAR1 blockade rescued HIV-1-induced CD4 ${ }^{+} \mathrm{T}$ cell depletion despite elevated HIV-1 replication, and exogenous IFN- $\alpha$ accelerated $\mathrm{CD}^{+} \mathrm{T}$ cell depletion (Figure $6 \mathrm{E}$ ), although it inhibited HIV-1 replication. In addition, inhibition of the activity of caspase- 3 by the specific inhibitor Z-DEVD-FMK also reduced HIV-1-induced $\mathrm{CD}^{+} \mathrm{T}$ cell apoptosis and rescued $\mathrm{CD}^{+}{ }^{+} \mathrm{T}$ cell number (Figure 6, F-H). In contrast, caspase-1 expression in $\mathrm{CD}^{+} \mathrm{T}$ cells did not increase in HIV-1infected samples (Supplemental Figure 4) and inhibition of caspase-1 activity did not prevent $\mathrm{CD}^{+} \mathrm{T}$ cell 
A
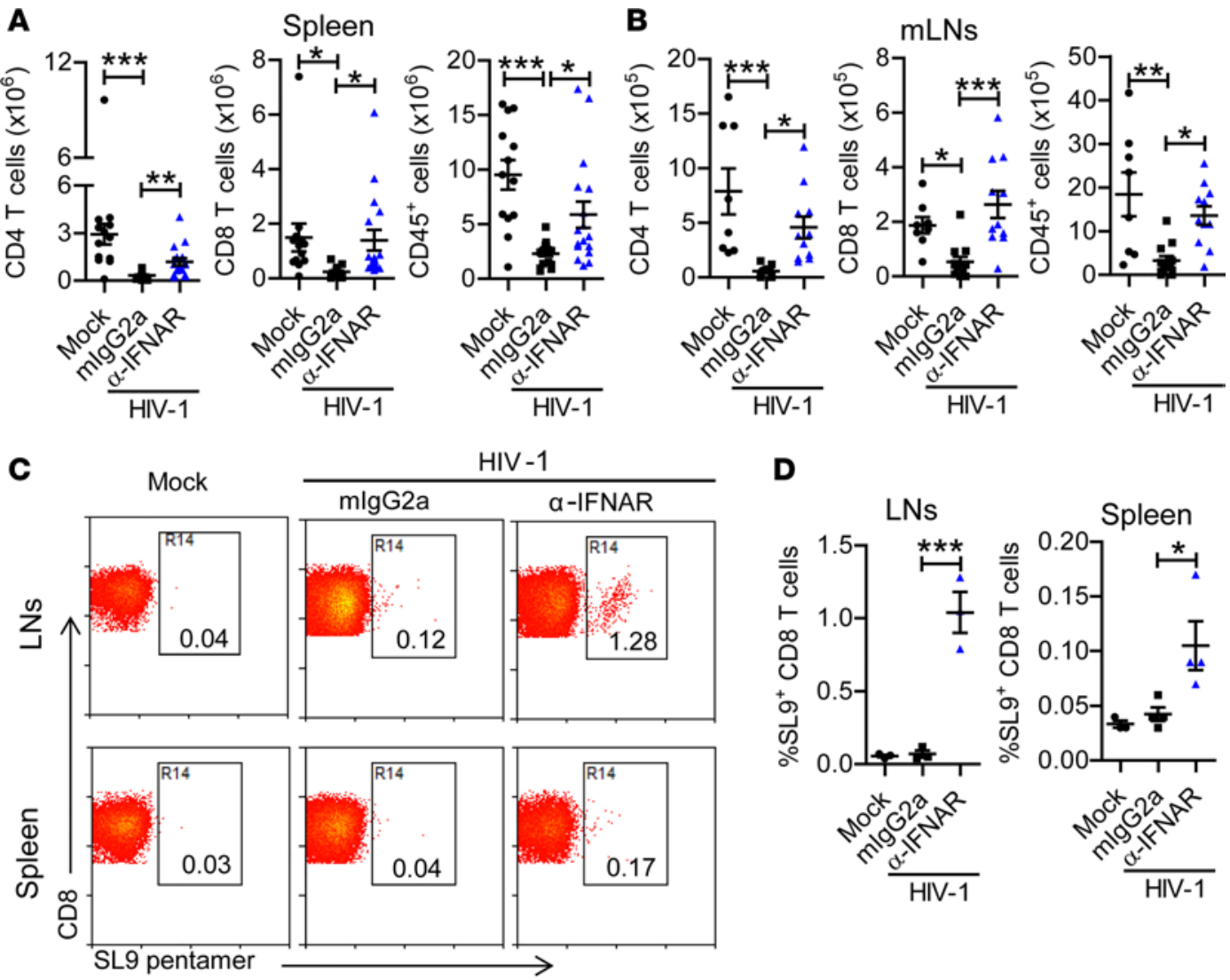

D

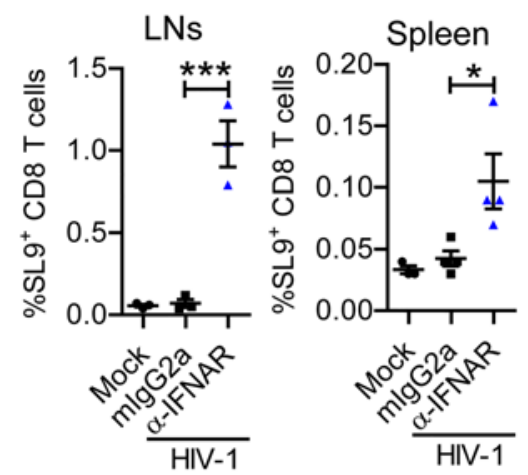

Figure 5. IFNAR1 blockade during persistent HIV-1 infection rescues human T cells and total human leukocytes in humanized mice. Humanized mice infected with HIV-1 were treated with a mAb against IFN- $\alpha / \beta$ receptor 1 (IFNAR1) or isotype control (mouse IgG2a) twice per week from 6 to 10 weeks postinjection (wpi). Mice were sacrificed at 10 wpi. (A and B) Numbers of CD4+ $T$ cells (CD3+CD8-), CD8 ${ }^{+}$T cells (CD3 ${ }^{+}$CD8 ${ }^{+}$) and total human leukocytes in spleens (A) (mock, $n=13$; HIV-1 + mlgG2a, $n=18$; HIV-1 + anti-IFNAR1, $n=17$, combined data from 5 independent experiments) and mesenteric lymph nodes (mLNs) (B) (mock, $n=8$; HIV-1 + mlgG2a, $n=12$; HIV-1 + anti-IFNAR1, $n=11$, combined data from 3 independent experiments with mean values \pm SEM). (C and D) Humanized NSG-A2 mice transplanted with hematopoietic stem cells from HLA-A2-matched donor were infected with HIV-1 and treated with anti-IFNAR1 mAb or isotype control (mouse IgG2a) twice per week from 6 to 10 wpi. Mice were sacrificed at 10 wpi. Representative dot plots (C) and summarized data (D) show the percentages of $C D 8^{+} \mathrm{T}$ cells specific for the HLA-A2/SL-9 pentamer (an HLA-A2-restricted epitope consisting of amino acids 77-85 of HIV-1 p17 protein) among CD8 ${ }^{+}$T cells from lymph nodes (mock, $n=3$; HIV-1 + mIgG2a, $n=3$; HIV-1 + anti-IFNAR1, $n=3$ ) and spleens (mock, $n=3$; HIV-1 + mIgG2a, $n=4$; HIV-1 + anti-IFNAR1, $n=4) .{ }^{*} P<0.05,{ }^{* *} P<0.01,{ }^{* * *} P<0.001$ by 1 -way ANOVA with Bonferroni's post hoc test.

depletion (Figure 6I). Taken together, these results indicate that sustained IFN-I signaling contributes to apoptosis of $\mathrm{CD}^{+} \mathrm{T}$ cells during persistent HIV-1 infection.

IFNAR1 blockade rescues function of human $T$ cells during persistent HIV-1 infection. We next analyzed the contribution of IFN-I signaling to HIV-1-induced functional impairment of T cells. We found that IFNAR1 blockade restored the ability of both $\mathrm{CD}^{+}$and $\mathrm{CD}^{+} \mathrm{T}$ cells to produce IFN- $\gamma$ and IL-2 upon PMA/ionomycin stimulation (Figure 7, A-C and Supplemental Figure 5A). IFNAR1 blockade did not reduce the expression level of $\mathrm{PD}-1$ on $\mathrm{T}$ cells (Supplemental Figure 6A), which was correlated with more viral replication (Supplemental Figure 6B). We further analyzed the function of HIV-1-specific T cells after IFNAR1 blockade. When stimulated with an HIV-1 Gag peptide pool ex vivo, both CD8 ${ }^{+}$ and $\mathrm{CD}^{+} \mathrm{T}$ cells from hu-mice with IFNAR1 blockade produced significantly higher levels of IFN- $\gamma$ and IL-2 (Figure 7, D-F and Supplemental Figure 5B), indicating that IFNAR1 blockade also rescued functional responses of HIV-1-specific T cells in persistently infected hosts. Taken together, these results show that IFNAR1 blockade during persistent HIV-1 infection rescues both the number and function of human T cells, including HIV-1-specific T cells.

\section{Discussion}

The contribution of IFN-I signaling to HIV-1 replication and immunopathogenesis during chronic infection is not clearly defined $(3,4)$, although persistent activation of plasmacytoid dendritic cells (pDCs) has been 
A

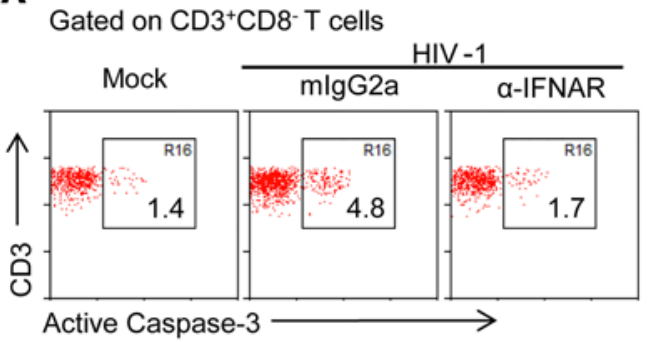

B

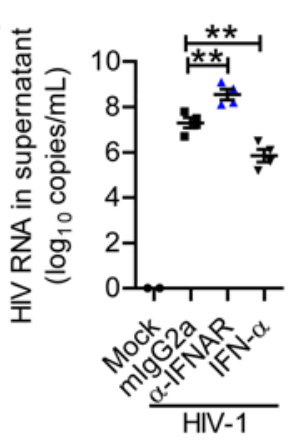

C

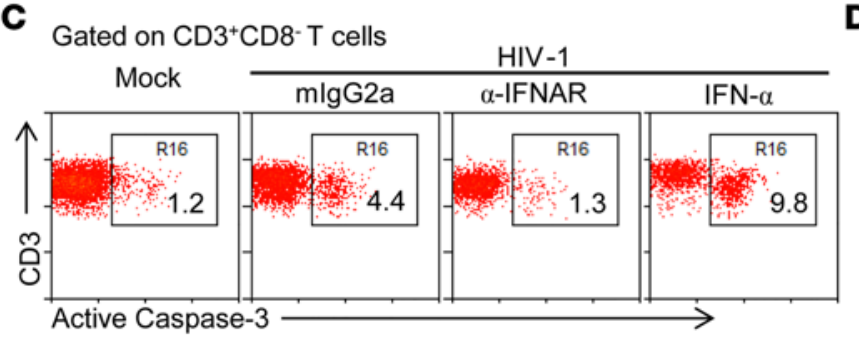

D E

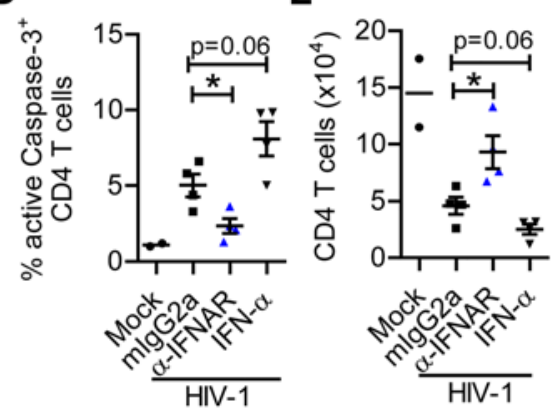

$\mathbf{F}$

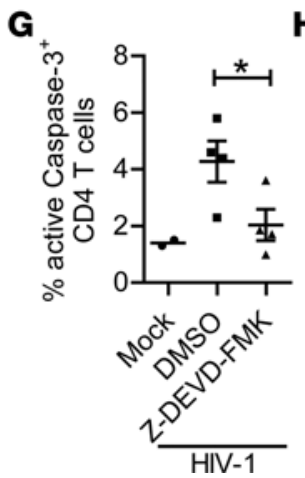

H I
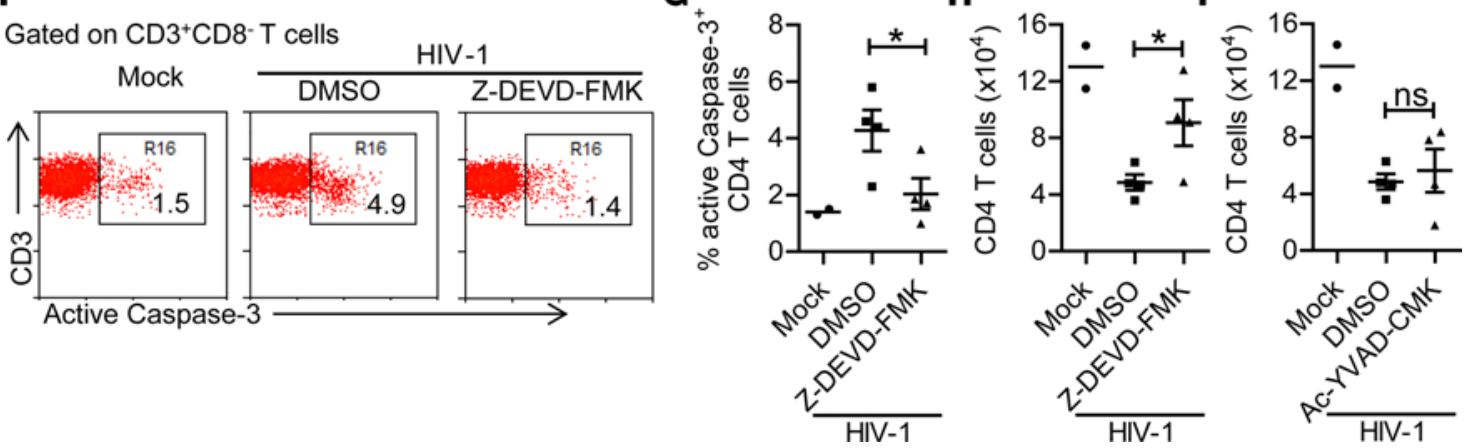

Figure 6. IFNAR1 blockade or inhibition of caspase-3 activity rescues CD4 ${ }^{+}$T cells from HIV-1-induced depletion. (A) Humanized mice infected with HIV-1 were treated with a mAb against IFN- $\alpha / \beta$ receptor 1 (IFNAR1) or isotype control (mouse IgG2a) twice per week from 6 to 10 weeks postinfection (wpi). Mice were sacrificed at 10 wpi. Representative dot plots and summarized data (mock, $n=6$; HIV-1 + mlgG2a, $n=9$; HIV-1 + anti-IFNAR1, $n=9$, combined data from 2 independent experiments with mean values \pm SEM) show the percentage splenic CD4+ $T$ cells expressing active caspase-3. (B-E) Splenocytes from mock $(n=$ 2) or HIV-1-infected $(n=4)$ humanized mice were cultured with IL-2 $(20 \mathrm{U} / \mathrm{ml})$ in vitro in the presence of control mlgG2a $(10 \mu \mathrm{g} / \mathrm{ml})$, anti-IFNAR1 $(10 \mu \mathrm{g} / \mathrm{ml})$ or IFN- $\alpha(200 \mathrm{U} / \mathrm{ml})$ for 10 days. At day 10 , the cells were counted and used for analysis. (B) HIV-1 RNA levels in culture supernatant. (C and D) Representative dot plots (C) and summarized data (D) show the percentages of $C D 4^{+} T$ cells with active caspase-3. (E) Number of live CD4 ${ }^{+} T$ cells in each group. (F-I) Splenocytes from mock $(n=2)$ or HIV-1-infected $(n=4)$ humanized mice were cultured with IL-2 in vitro in the presence of DMSO or caspase-3 inhibitor Z-DEVD-FMK or caspase-1 inhibitor Ac-YVAD-CMK for 10 days. At day 10, the cells were counted and used for staining. (F and $\mathbf{G})$ Representative dot plots (F) and summarized data (C) show the percentages of active-caspase- $3^{+} C D 4^{+} T$ cells. ( $H$ and I) Number of live $C D 4^{+} T$ cells in each group. Data are representative of 2 independent experiments with mean values $\pm \mathrm{SEM}$ ). ${ }^{*} P<0.05,{ }^{* *} P<0.01$ by unpaired, 2 -tailed Student's $t$ test to compare differences between 2 groups. ns, not significant.

reported to contribute to HIV-induced immunopathogenesis $(37,38)$. Using an anti-IFNAR1 mAb to block IFN-I signaling in vivo, we showed here that IFNAR1 blockade during persistent HIV-1 infection increased HIV-1 replication in hu-mice. We discovered that IFNAR1 blockade rescued human T cells numerically and functionally despite elevated HIV-1 replication and T cell activation. Our results agree with recent findings showing that persistent IFN-I signaling plays a detrimental role during chronic LCMV infection and blocking IFN-I signaling with an anti-IFNAR mAb could reverse $\mathrm{T}$ cell exhaustion and enhance antiviral immune responses $(16,17)$.

During the chronic phase of HIV-1 infection, a positive correlation between the expression of ISGs and HIV-1 viremia has been reported $(6,19)$. Persistent ISG expression is also associated with pathogenic SIV infection $(12,13,15,39)$. However, the role of IFN-I signaling in HIV-1 infection and 
A

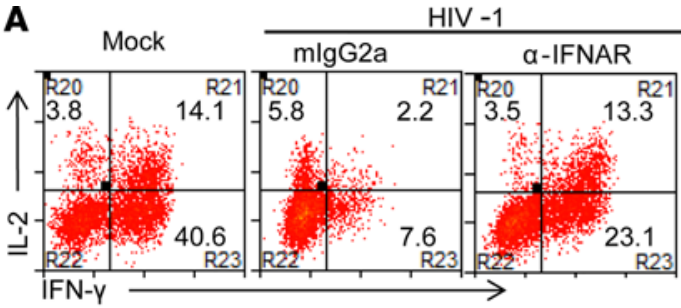

B
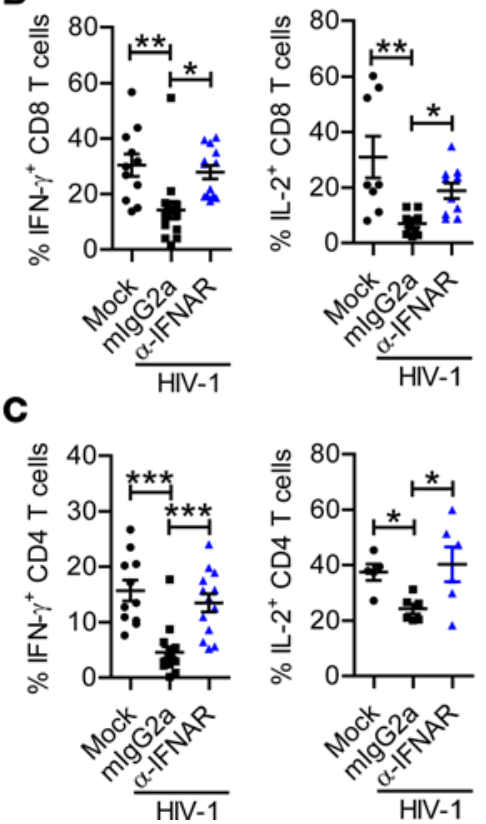
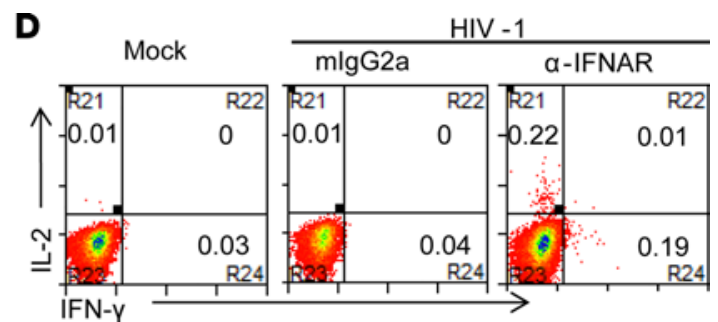

E
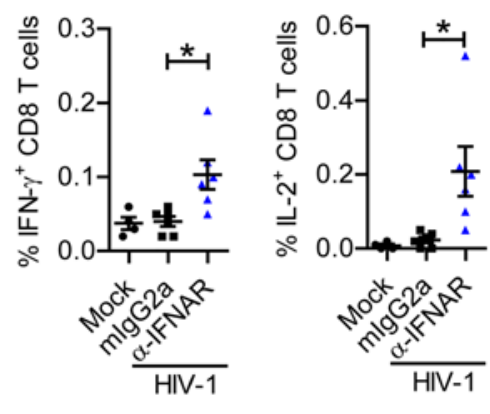

$\mathbf{F}$

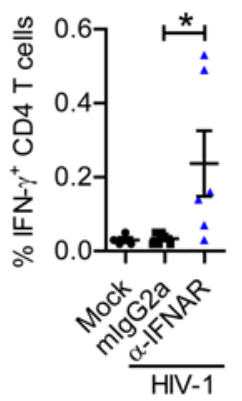

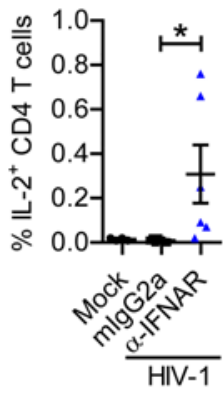

Figure 7. IFNAR1 blockade during persistent HIV-1 infection rescues the function of human T cells, including HIV-1-specific T cells. Humanized mice infected with HIV-1 were treated with a mAb against IFN- $\alpha / \beta$ receptor 1 (IFNAR1) or isotype control (mouse IgG2a) twice per week from 6 to 10 weeks postinfection (wpi). Mice were sacrificed at 10 wpi. (A-C) Splenocytes were stimulated ex vivo with PMA plus ionomycin for 4 hours followed by intracellular cytokine staining. Representative dot plots (A) and summarized data show the percentages of CD $8^{+} T$ cells (B) and CD4 ${ }^{+} T$ cells $(\mathbf{C})$ producing IFN- $\gamma$ (mock, $n=11$; HIV-1 + mIgG2a, $n=15$; HIV-1 + anti-IFNAR1, $n=13$, combined data from 4 independent experiments with mean values \pm SEM) or IL-2 (mock, $n=8$; HIV-1 + mIgG2a, $n=10$; HIV-1 + anti-IFNAR1, $n=10$, combined data from 3 independent experiments with mean values \pm SEM). (D-F) Splenocytes were stimulated ex vivo with peptide pools of HIV-1 Gag protein for 8 hours (brefeldin A was added at 3 hours) followed by intracellular cytokine staining. Representative dot plots (D) and summarized data show the percentages of CD8 ${ }^{+} \mathrm{T}$ cells (E) and CD4 ${ }^{+}$T cells (F) producing IFN- $\gamma$ and IL-2 (mock, $n=4$; HIV-1 + mIgC2a, $n=6$; HIV-1 + anti-IFNAR1, $n=6$, combined data from 2 independent experiment with mean values \pm SEM). ${ }^{*} P<0.05,{ }^{* *} P<$ $0.01,{ }^{* *} P<0.001$ by 1 -way ANOVA and Bonferroni's post hoc test.

disease progression is not clearly defined. We found that blocking endogenous IFN-I signaling during persistent HIV-1 infection by anti-IFNAR1 mAb increased viral replication. This result is consistent with our previous finding that depletion of pDCs, the major IFN-I-producing cells during HIV-1 infection, increased HIV-1 replication (38). Thus, we demonstrate that HIV-1 is still sensitive to IFN-I during persistent HIV-1 infection.

HIV-1 disease progression is associated with depletion of human leukocytes including human $\mathrm{CD} 4^{+} \mathrm{T}$ cells. Although several reports show that persistent ISG induction is correlated with disease progression in HIV-1-infected patients (19-21), the direct causal link between IFN-I signaling and $\mathrm{CD} 4^{+} \mathrm{T}$ cell depletion is not clearly established. In the present study, we found that IFNAR1 blockade rescued both the number and function of human $\mathrm{T}$ cells, and prevented HIV-1-induced $\mathrm{CD}^{+} \mathrm{T}$ cell apoptosis, in spite of higher levels of virus replication. Thus, sustained IFN-I signaling plays a major role in $\mathrm{CD} 4^{+} \mathrm{T}$ cell depletion during persistent HIV-1 infection. Interestingly, we found that IFNAR1 blockade increased the expression of activation markers CD38/HLA-DR and Ki-67 on both $\mathrm{CD} 4^{+}$and $\mathrm{CD} 8^{+} \mathrm{T}$ cells and was positively correlated with HIV-1 viremia. These data also indicate that IFN-Is are not essential for HIV-induced aberrant immune activation. In the absence of IFN-I signaling, higher levels of HIV-1 viremia or aberrant immune activation failed to lead to $\mathrm{CD} 4^{+} \mathrm{T}$ cell depletion. We thus conclude that IFN-Is are critical to the death of human T cells during chronic HIV-1 infection. 
IFN-Is also play an important role in modulating T cell function (40). During chronic LCMV infection in mice, blocking IFN-I signaling with an anti-IFNAR1 $\mathrm{mAb}$ can enhance antiviral immune responses and prevent or accelerate clearance of persistent LCMV infection in mice $(16,17)$. We demonstrate here that during chronic HIV-1 infection in hu-mice, blocking IFN-I signaling rescued the HIV-1-specific T cell function. IFNAR blockade may lead to the improvement in anti-HIV-1 $\mathrm{T}$ cell responses by several mechanisms. First, it has been reported that sustained IFN-I signaling leads to the expression of the negative immune regulators IL-10 and PD-L1 on antigen-presenting cells during LCMV infection in mice. Blockade of IFN-I signaling improves DC functions and may contribute to the restoration of anti-LCMV $\mathrm{T}$ cell responses $(16,17,40)$. Second, IFNAR signaling in $\mathrm{T}$ cells may have direct antiproliferative and proapoptotic effects $(36,40-42)$. In addition, the preservation of helper CD4 ${ }^{+} \mathrm{T}$ cells by blocking IFNAR may also help to enhance anti-HIV-1 CD8 ${ }^{+} \mathrm{T}$ cell activity. However, blockade of IFNAR in hu-mice also led to elevated HIV-1 replication. This may be due to the loss of an innate anti-HIV-1 effect of IFN-Is after IFNAR blocking that overcomes the benefit of reversed $\mathrm{T}$ cell response in viral inhibition. It is also important to point out that the human immunity developed in hu-mice is not fully functional, as found in immunocompetent hosts $(24,25)$. The restored anti-HIV-1 T cell immune response by IFNAR blockade in hu-mice may not be sufficiently robust to control HIV-1 replication.

A recent report showed that blockade of IFNAR2 during the chronic phase of HIV-1 infection in hu-mice led to decreased viral replication, diminished HIV-1-driven immune activation, and restored HIV$1-$ specific $\mathrm{CD}^{+} \mathrm{T}$ cell function (43). The difference between the 2 studies is that Zhen et al. used a mAb to block IFNAR2, while we used a mAb to block IFNAR1. As reported previously, IFN- $\beta$ can specifically and uniquely bind to IFNAR1 to initiate downstream signaling in an IFNAR2-independent manner (44). It is thus likely that blocking IFNAR1, but not IFNAR2, can completely block all types of IFN-I signaling. The remaining IFN-I signaling that cannot be blocked by anti-IFNAR2 $\mathrm{mAb}$ in the report by Zhen et al. may still contribute to the suppression of HIV-1 replication. Interestingly, HIV-specific CD8 ${ }^{+} \mathrm{T}$ cell function was restored either by IFNAR1 blockade or by IFNAR2 blockade. The reversed anti-HIV-1 T cell function may synergize with the remaining IFN-I signaling to further control HIV-1 replication (43). Thus, it will be important to dissect the specific IFN-I subtypes, their signaling pathways, and ISGs that contribute to viral inhibition and host immunity impairment during persistent HIV-1 infection.

During the acute phase (0-4 wpi) of SIV infection, blocking IFN-I signaling with IFN- $\alpha 2 / \mathrm{IFN}$-ant, with increased IFNAR2 binding but diminished IFNAR1 binding activity (22), leads to elevated SIV replication and accelerated disease progression in rhesus monkeys (7). In the same study, they report that, while administration of PEG-IFN- $\alpha 2$ a during preinfection and acute infection results in decreased SIV transmission, continued IFN- $\alpha 2$ a treatment appears to induce IFN-I desensitization and to decrease antiviral gene expression, also resulting in increased SIV replication and accelerated CD4 ${ }^{+} \mathrm{T}$ cell loss (7). This study has at least one major difference from our study in that IFN-I signaling was blocked only during acute SIV infection. In the absence of IFN-I blocking during the late stage of infection, the higher levels of SIV infection (probably also with higher IFN-I activity) may lead to the accelerated disease progression. It is generally believed that chronic IFN-I signaling during persistent infection can lead to general immune suppression (40). Thus, IFN-I signaling is beneficial during the acute phase to inhibit HIV-1 infection and to prime host immune responses, but becomes harmful during the chronic phase of infection. Blocking IFN-I signaling with either the anti-INFAR1 or anti-IFNAR2 $\mathrm{mAb}$ in rhesus monkeys during chronic SIV infection will be of great interest to further clarify these findings.

Interestingly, a TLR7 and TLR9 antagonist in SIV-infected monkeys inhibits IFN-I production by pDCs but does not affect viral load, immune activation, and $\mathrm{CD}^{+} \mathrm{T}$ cell loss (45). This suggests that cells other than pDCs, including myeloid DCs (mDCs), may also produce IFN-Is during persistent SIV infection. The TLR7 and TLR 9 antagonist fails to block IFN-I production by mDCs (45). Thus, IFN-Is induced by SIV infection in pDCs and other cell types may contribute to the pathogenesis of SIV infection.

In summary, we report that IFN-Is function as a double-edged sword during persistent HIV-1 infection. They are beneficial by inhibiting HIV-1 replication but detrimental by inducing $\mathrm{T}$ cell depletion and dysfunction. In HIV-1-infected patients, despite efficient suppression of HIV-1 replication with combined antiretroviral therapy, slightly elevated levels of IFN-Is and ISGs persist in some individuals (46, 47), which may impede immune recovery and foster viral persistence $(4,48)$. Our recent study indicates that blocking IFN-I signaling during antiretroviral therapy enhances T cell recovery and reduces HIV-1 reservoirs (23). It is conceivable that blocking IFNAR may provide a novel approach to facilitate recovery 
of functional anti-HIV-1 immune responses, thereby enabling control of HIV-1 reservoirs to achieve an HIV-1 cure, as well as to treat those immune nonresponder patients with elevated IFN-I signaling despite effective antiretroviral therapy.

\section{Methods}

Construction of hu-mice. NRG (NOD-Rag2-/- $\gamma \mathrm{c}^{-/-}$) and NSG-A2 (NOD.Cg-Prkdc ${ }^{\text {scid }} \gamma \mathrm{c}^{-/-}$Tg (HLA-A2.1) mice were obtained from the Jackson Laboratory. Humanized NRG/NSG-A2 mice with a functional human immune system were generated by intrahepatic injection of newborn mice with human fetal liver-derived $\mathrm{CD}^{+} 4^{+}$hematopoietic progenitor cells as previously reported (38). Humanized bone marrow/liver/thymus (BLT) mice were generated as previously reported (26). Briefly, 6- to 8-week-old NRG mice were sublethally irradiated and anesthetized, and $\sim 1-\mathrm{mm}^{3}$ fragments of human fetal thymus were implanted under the kidney capsule. CD $34^{+}$hematopoietic progenitor cells purified from fetal liver of the same donor were injected i.v. within 3 hours. Human immune cell engraftment was detected by flow cytometry 10-12 weeks after transplantation. All mice were housed and bred in a specific pathogen-free environment.

HIV-1 infection of hu-mice. The CCR5-tropic strain of HIV-1 (JR-CSF) was generated by transfection of 293T cells (ATCC) with plasmid containing full-length HIV-1 (JR-CSF) genome. Hu-mice with stable human leukocyte reconstitution were anesthetized and infected with HIV-1 (JR-CSF) (10 ng p24/mouse) through retro-orbital injection. Hu-mice infected with $293 \mathrm{~T}$ supernatant were used as mock control groups.

Development of anti-IFNAR1 $m A b$. The generation of the anti-IFNAR1 mAb was performed as reported (23). Briefly, the mouse cell line L-929 transfected with the human IFNAR1 (extracellular domain and transmembrane domain) expression plasmid was used as the immunogen for immunization with CpG1826 as adjuvant. After 5 immunizations, the spleen cells were fused with the mouse myeloma cell line SP2/0. 293T cells transfected with the human IFNAR1 expression plasmid were used for screening the clones that could secret the IFNAR1-binding antibody by flow cytometry. Then, an IFN-I reporter 293T cell line, which had been stably transfected with a mouse Mx2 promoter-driven EGFP, was used to screen $\mathrm{mAb}$ clones that could block the human IFNAR1 signaling.

In vivo IFNAR1-blocking $m A b$ treatments. To confirm the in vivo neutralizing activity of the $\mathrm{mAb}$, hu-mice were treated i.p. with $\mathrm{mAb} 6$ hours prior to R 848 treatment. To block IFN-I signaling during chronic HIV-1 infection, hu-mice were treated i.p. with IFNAR1-blocking mAbs twice per week with $400 \mu \mathrm{g} / \mathrm{mouse}$ at the first injection and $200 \mu \mathrm{g} /$ mouse for the following treatments from 6 to $10 \mathrm{wpi}$. Cohorts of mice were randomized into different treatment groups by the level of HIV-1 RNA in plasma.

Quantification of mRNA expressionbyreal-timereversetranscription PCR. RNA from PBMCs or whole splenocytes from hu-mice was isolated with the RNeasy Plus extraction kit (Qiagen) and converted to cDNA by reverse transcription with random hexamers and SuperScript III First-Strand Synthesis (Invitrogen). cDNA was then analyzed by real-time PCR using gene-specific primers for ISG15 (5'-CGCAGATCACCCAGAAGATCG-3' and 5'-TTCGTCGCATTTGTCCACCA-3'), MxA (5'-GGTGGTCCCCAGTAATGTGG-3' and 5'-CGTCAAGATTCCGATGGTCCT-3'), OAS1 (5'-TGTCCAAGGTGGTAAAGGGTG-3' and 5'-CCGGCGATTTAACTGATCCTG-3'), IFITM3 (5'-ATGTCGTCTGGTCCCTGTTC-3' and 5'-GTCATGAGGATGCCCAGAAT-3'), Mx2 (5'-CAGAGGCAGCGGAATCGTAA-3' and 5'-TGAAGCTCTAGCTCGGTGTTC-3'), Trim22 (5'-CTGTCCTGTGTGTCAGACCAG-3' and 5'-TGTGGGCTCATCTTGACCTCT-3'), and IRF7 (5'-GCTGGACGTGACCATCATGTA-3' and 5'-GGGCCGTATAGGAACGTGC-3'). The ISG expression levels were normalized to human CD45 controls. Results were calculated as a fold change in gene expression, relative to mock mice using the deltadelta Ct method of analysis (49).

Flow cytometry. FITC-conjugated anti-human HLA-DR (L243), IFN- $\gamma$ (4S.B3), PE-conjugated anti-human CD38 (HIT2), PerCP/Cy5.5-conjugated anti-human CD4 (RPA-T4), PE/Cy7-conjugated anti-human CD8 (HIT8a), IL-2 (MQ1-17H12), BV421-conjugated anti-human PD-1 (EH12.2H7), APC-conjugated anti-human Ki-67 (Ki-67), and APC/Cy7-conjugated anti-human CD45 (HI30) were purchased from Biolegend. FITC-conjugated anti-HIV-1 p24 was purchased from Beckman Coulter. PE-conjugated anti-human active caspase-3 (C92-605) was purchased from BD Pharmingen. Pacific orange-conjugated anti-mouse CD45 (30-F11), PE/Texas red-conjugated anti-human CD3 (7D6), and a LIVE/DEAD Fixable Yellow Dead Cell Stain Kit were purchased from Invitrogen. PE-conjugated A*02:01/SLYNTVATL pentamer was purchased from PROIMMUNE. 
For surface staining, single-cell suspensions prepared from peripheral blood, spleen, or mLNs of hu-mice were stained with surface markers and analyzed on a CyAn ADP flow cytometer (Dako). For intracellular staining, cells were first stained with surface markers, and then fixed and permeabilized with Cytofix/Cytoperm buffer (BD Bioscience), followed by intracellular staining. PE-conjugated anti-human active caspase-3 (C92-605) was used to detect intracellular activation of caspase-3. Fluorescently labeled inhibitors of caspase-1 (FLICA 660 Caspase-1 Assay Kit, ImmunoChemistry Technologies) were used to determine intracellular activation of caspase-1. Data were analyzed using Summit4.3 software (Dako).

T cell stimulation and intracellular cytokine staining. For nonspecific stimulation, splenocytes from hu-mice were stimulated ex vivo with PMA $(50 \mathrm{ng} / \mathrm{ml})$ and ionomycin $(1 \mu \mathrm{M})$ (Sigma-Aldrich) for 4 hours in the presence of brefeldin A (Biolegend). For antigen-specific stimulation, splenocytes from hu-mice were stimulated ex vivo with peptide pools ( $2 \mu \mathrm{g} / \mathrm{ml}$ for each peptide) for HIV-1 GAG protein [PepMix HIV (GAG) Ultra, JPT Innovation Peptide Solutions] for 3 hours without brefeldin A and then 5 hours in the presence of brefeldin A. Cells were then fixed and permeabilized with Cytofix/Cytoperm buffer, and intracellular staining was then performed.

HIV-1 genomic RNA detection in plasma. HIV-1 RNA was purified from plasma with the QIAamp Viral RNA Mini Kit (Qiagen). The RNA was then reverse transcribed and quantitatively detected by real-time PCR using the TaqMan Fast Virus 1-Step PCR kit (ThermoFisher Scientific). The primers used for detecting the HIV Gag gene were 5'-GGTGCGAGAGCGTCAGTATTAAG-3' and 5'-AGCTCCCTGCTTGCCCATA-3'. The probe FAM-AAAATTCGGTTAAGGCCAGGGGGAAAGAA-QSY7 used for detection was ordered from Applied Biosystems and the reactions were set up according to the manufacturer's guidelines and were run on the QuantStudio 6 Flex PCR system (Applied Biosystems).

Detection of cytokines in plasma. Human pan IFN- $\alpha$ (subtypes $1 / 13,2,4,5,6,7,8,10,14,16$, and 17) were detected by ELISA using human IFN- $\alpha$ pan ELISA kits purchased from Mabtech. Human IFN- $\beta$ was detected by ELISA using the Verikine-HS Human Interferon Beta Serum ELISA kit. Human IFN- $\gamma$, TNF- $\alpha$, IP-10, and MCP-1 in plasma of hu-mice were detected by Luminex immunology multiplex assay (Millipore).

Ex vivo assays. Splenocytes from mock or persistently HIV-1 infected (15-20 wpi) hu-mice were cultured with $20 \mathrm{U} / \mathrm{ml}$ of IL-2 (NIH AIDS Reagent Program) ex vivo in the presence of murine IgG2a control $(10 \mu \mathrm{g} / \mathrm{ml}$, Bio X Cell), anti-IFNAR1 $(10 \mu \mathrm{g} / \mathrm{ml})$, or IFN- $\alpha(200 \mathrm{U} / \mathrm{ml}$, PBL Assay Science), caspase-3 inhibitor Z-DEVD-FMK (10 $\mu \mathrm{M})$, caspase-1 inhibitor Ac-YVAD-CMK (50 $\mu \mathrm{M})$ (both Enzo Life Sciences), or DMSO (Sigma-Aldrich) as control for 10 days. Media were half-changed every 2 to 3 days. At day 10, the cells were counted and used for staining.

Statistics. In all experiments, significance levels of data were determined by using Prism5 (GraphPad Software). Experiments were analyzed by 2-tailed Student's $t$ test, or by 1-way ANOVA and Bonferroni's post hoc test according to the assumptions of the test, as indicated for each experiment. Correlations between variables were evaluated using the Spearman rank correlation test. A $P$ value less than 0.05 was considered significant. The number of animals and replicates is specified in each figure legend.

Study approval. Human fetal liver and thymus (gestational age of 16 to 20 weeks) were obtained from medically or elective indicated termination of pregnancy through a nonprofit intermediary working with outpatient clinics (Advanced Bioscience Resources). Written informed consent of the maternal donors was obtained in all cases, under regulations governing the clinic. The project was reviewed by the University's Office of Human Research Ethics, which has determined that this submission does not constitute human subjects research as defined under federal regulations [45 CFR 46.102(d or f) and 21 CFR 56.102(c), (e), and (l)]. All animal studies were approved by the University of North Carolina Institutional Animal Care and Use Committee (IACUC ID: 14-100) and were conducted following NIH guidelines for housing and care of laboratory animals.

\section{Author contributions}

L. Cheng, LZ, and LS conceived and designed the study. L. Cheng, HY, GL, FL, JM, JL, and L. Chi performed experiments. L. Cheng, LZ, and LS analyzed and interpreted the data and prepared the manuscript.

\section{Acknowledgments}

We thank Yaxu Wu for technical support, and the Lineberger Comprehensive Cancer Center cores, UNC flow cytometer core, DLAM and UNC CFAR for support. This study was supported in part by NIH grants AI127346, AI095097, DK095962, and MOH 2017ZX10202101-004 to L.S. and a grant from the Beijing Municipal Science and Technology Commission (D171100000517002) to L.Z. 
Address correspondence to: Lishan Su, Room 5008, Masico Hall, 125 Mason Farm Road, Chapel Hill, North Carolina 27514, USA. Phone: 919.966.6654; Email: lishan_su@med.unc.edu. Or to: Liguo Zhang, 15 Datun Road, Chaoyang District, Beijing, China, 100101. Phone: 010.64862568; Email: liguozhang@ ibp.ac.cn. Or to: Liang Cheng, Room 5229N, Masico Hall, 125 Mason Farm Road, Chapel Hill, North Carolina 27514, USA. Phone: 919.966.4913; Email: cheng184@email.unc.edu.

1. Zuniga EI, Hahm B, Oldstone MB. Type I interferon during viral infections: multiple triggers for a multifunctional mediator. Curr Top Microbiol Immunol. 2007;316:337-357.

2. Schoggins JW, et al. A diverse range of gene products are effectors of the type I interferon antiviral response. Nature. 2011;472(7344):481-485.

3. Doyle T, Goujon C, Malim MH. HIV-1 and interferons: who's interfering with whom? Nat Rev Microbiol. 2015;13(7):403-413.

4. Bosinger SE, Utay NS. Type I interferon: understanding its role in HIV pathogenesis and therapy. Curr HIV/AIDS Rep. 2015;12(1):41-53

5. Stacey AR, et al. Induction of a striking systemic cytokine cascade prior to peak viremia in acute human immunodeficiency virus type 1 infection, in contrast to more modest and delayed responses in acute hepatitis B and C virus infections. $J$ Virol. 2009;83(8):3719-3733.

6. Hardy GA, et al. Interferon- $\alpha$ is the primary plasma type-I IFN in HIV-1 infection and correlates with immune activation and disease markers. PLoS One. 2013;8(2):e56527.

7. Sandler NG, et al. Type I interferon responses in rhesus macaques prevent SIV infection and slow disease progression. Nature. 2014;511(7511):601-605.

8. Lavender KJ, et al. Interferon alpha subtype-specific suppression of HIV-1 infection in vivo. J Virol. 2016;90(13):6001-6013.

9. Abraham S, Choi JG, Ortega NM, Zhang J, Shankar P, Swamy NM. Gene therapy with plasmids encoding IFN- $\beta$ or IFN- $\alpha 14$ confers long-term resistance to HIV-1 in humanized mice. Oncotarget. 2016;7(48):78412-78420.

10. Parrish NF, et al. Phenotypic properties of transmitted founder HIV-1. Proc Natl Acad Sci U S A. 2013;110(17):6626-6633.

11. Fenton-May AE, et al. Relative resistance of HIV-1 founder viruses to control by interferon-alpha. Retrovirology. 2013;10:146.

12. Bosinger SE, et al. Global genomic analysis reveals rapid control of a robust innate response in SIV-infected sooty mangabeys. $J$ Clin Invest. 2009;119(12):3556-3572.

13. Jacquelin B, et al. Nonpathogenic SIV infection of African green monkeys induces a strong but rapidly controlled type I IFN response. J Clin Invest. 2009;119(12):3544-3555.

14. Harris LD, et al. Downregulation of robust acute type I interferon responses distinguishes nonpathogenic simian immunodeficiency virus (SIV) infection of natural hosts from pathogenic SIV infection of rhesus macaques. $J$ Virol. 2010;84(15):7886-7891.

15. Favre D, et al. Critical loss of the balance between Th17 and T regulatory cell populations in pathogenic SIV infection. PLoS Pathog. 2009;5(2):e1000295.

16. Wilson EB, et al. Blockade of chronic type I interferon signaling to control persistent LCMV infection. Science. 2013;340(6129):202-207.

17. Teijaro JR, et al. Persistent LCMV infection is controlled by blockade of type I interferon signaling. Science. 2013;340(6129):207-211.

18. Ng CT, et al. Blockade of interferon beta, but not interferon alpha, signaling controls persistent viral infection. Cell Host Microbe. 2015;17(5):653-661.

19. Rotger M, et al. Genome-wide mRNA expression correlates of viral control in CD4 ${ }^{+}$T-cells from HIV-1-infected individuals. PLoS Pathog. 2010;6(2):e1000781.

20. Hyrcza MD, et al. Distinct transcriptional profiles in ex vivo $\mathrm{CD} 4^{+}$and $\mathrm{CD} 8^{+} \mathrm{T}$ cells are established early in human immunodeficiency virus type 1 infection and are characterized by a chronic interferon response as well as extensive transcriptional changes in CD8 ${ }^{+}$T cells. J Virol. 2007;81(7):3477-3486.

21. Sedaghat AR, et al. Chronic CD4 ${ }^{+} \mathrm{T}$-cell activation and depletion in human immunodeficiency virus type 1 infection: type I interferon-mediated disruption of T-cell dynamics. J Virol. 2008;82(4):1870-1883.

22. Levin D, et al. Multifaceted activities of type I interferon are revealed by a receptor antagonist. Sci Signal. 2014;7(327):ra50.

23. Cheng L, et al. Blocking type I interferon signaling enhances T cell recovery and reduces HIV-1 reservoirs. J Clin Invest. 2017;127(1):269-279.

24. Shultz LD, Brehm MA, Garcia-Martinez JV, Greiner DL. Humanized mice for immune system investigation: progress, promise and challenges. Nat Rev Immunol. 2012;12(11):786-798.

25. Zhang L, Su L. HIV-1 immunopathogenesis in humanized mouse models. Cell Mol Immunol. 2012;9(3):237-244

26. Namikawa R, Kaneshima H, Lieberman M, Weissman IL, McCune JM. Infection of the SCID-hu mouse by HIV-1. Science. 1988;242(4886):1684-1686.

27. Buimovici-Klein E, Lange M, Klein RJ, Cooper LZ, Grieco MH. Is presence of interferon predictive for AIDS? Lancet. 1983;2(8345):344.

28. Kane M, et al. MX2 is an interferon-induced inhibitor of HIV-1 infection. Nature. 2013;502(7472):563-566.

29. Goujon C, et al. Human MX2 is an interferon-induced post-entry inhibitor of HIV-1 infection. Nature. 2013;502(7472):559-562.

30. Lu J, Pan Q, Rong L, He W, Liu SL, Liang C. The IFITM proteins inhibit HIV-1 infection. J Virol. 2011;85(5):2126-2137.

31. Yu J, et al. IFITM proteins restrict HIV-1 infection by antagonizing the envelope glycoprotein. Cell Rep. 2015;13(1):145-156

32. Paiardini M, Müller-Trutwin M. HIV-associated chronic immune activation. Immunol Rev. 2013;254(1):78-101.

33. Moir S, Chun TW, Fauci AS. Pathogenic mechanisms of HIV disease. Annu Rev Pathol. 2011;6:223-248.

34. Finkel TH, et al. Apoptosis occurs predominantly in bystander cells and not in productively infected cells of HIV- and SIVinfected lymph nodes. Nat Med. 1995;1(2):129-134. 
35. Herbeuval JP, et al. CD4 ${ }^{+}$T-cell death induced by infectious and noninfectious HIV-1: role of type 1 interferon-dependent, TRAIL/DR5-mediated apoptosis. Blood. 2005;106(10):3524-3531.

36. Fraietta JA, et al. Type I interferon upregulates Bak and contributes to T cell loss during human immunodeficiency virus (HIV) infection. PLoS Pathog. 2013;9(10):e1003658.

37. Zhang Z, et al. Plasmacytoid dendritic cells promote HIV-1-induced group 3 innate lymphoid cell depletion. J Clin Invest. 2015;125(9):3692-3703.

38. Li G, et al. Plasmacytoid dendritic cells suppress HIV-1 replication but contribute to HIV-1 induced immunopathogenesis in humanized mice. PLoS Pathog. 2014;10(7):e1004291.

39. Lederer S, et al. Transcriptional profiling in pathogenic and non-pathogenic SIV infections reveals significant distinctions in kinetics and tissue compartmentalization. PLoS Pathog. 2009;5(2):e1000296.

40. Crouse J, Kalinke U, Oxenius A. Regulation of antiviral T cell responses by type I interferons. Nat Rev Immunol. 2015;15(4):231-242.

41. Dondi E, Rogge L, Lutfalla G, Uzé G, Pellegrini S. Down-modulation of responses to type I IFN upon T cell activation. J Immunol. 2003;170(2):749-756

42. Bromberg JF, Horvath CM, Wen Z, Schreiber RD, Darnell JE. Transcriptionally active Stat1 is required for the antiproliferative effects of both interferon alpha and interferon gamma. Proc Natl Acad Sci U S A. 1996;93(15):7673-7678.

43. Zhen A, et al. Targeting type I interferon-mediated activation restores immune function in chronic HIV infection. J Clin Invest. 2017;127(1):260-268.

44. de Weerd NA, et al. Structural basis of a unique interferon- $\beta$ signaling axis mediated via the receptor IFNAR1. Nat Immunol. 2013;14(9):901-907.

45. Kader M, et al. Blocking TLR7- and TLR9-mediated IFN- $\alpha$ production by plasmacytoid dendritic cells does not diminish immune activation in early SIV infection. PLoS Pathog. 2013;9(7):e1003530.

46. Fernandez S, et al. CD4 ${ }^{+}$T-cell deficiency in HIV patients responding to antiretroviral therapy is associated with increased expression of interferon-stimulated genes in CD4+ T cells. J Infect Dis. 2011;204(12):1927-1935

47. Dunham RM, et al. Discordance between peripheral and colonic markers of inflammation during suppressive ART. $J$ Acquir Immune Defic Syndr. 2014;65(2):133-141.

48. Deeks SG. HIV infection, inflammation, immunosenescence, and aging. Annu Rev Med. 2011;62:141-155.

49. Schmittgen TD, Livak KJ. Analyzing real-time PCR data by the comparative C (T) method. Nat Protoc. 2008;3(6):1101-1108. 